\title{
Anisotropy of Cosmic Ray Fluxes measured with AMS-02 on the ISS
}

\section{J. Casaus ${ }^{* \dagger}$, C. Maña, M. Molero, M.A. Velasco}

Centro de Investigaciones Energéticas, Medioambientales y Tecnológicas (CIEMAT)

E-mail: Jorge. Casaus@ciemat.es

\section{Gebauer, M. Graziani}

Institut für Experimentelle Teilchenphysik, Karlsruhe Institute of Technology (KIT)

\section{G. La Vacca, M. Gervasi, P.G. Rancoita}

INFN and Universita' di Milano-Bicocca

A measurement of the dipole anisotropy in galactic coordinates for individual cosmic ray species has been performed with the Alpha Magnetic Spectrometer (AMS) onboard the International Space Station (ISS). Results are presented for the first 7.5 years of data taking for protons, helium, carbon and oxygen, and for 6.5 years for positrons and electrons. Thanks to its wide field of view and to the inclination of the ISS orbit, AMS provides nearly full sky coverage in galactic coordinates, which allows to determine the three independent dipole components. The results are consistent with isotropy for all particle species and upper limits on the dipole amplitude have been derived. For energies above $16 \mathrm{GeV}$ a limit of $\delta<1.9 \%$ and $\delta<0.5 \%$ at the $95 \%$ C.I. has been obtained for positrons and electrons, respectively. For protons, helium, carbon and oxygen, upper limits of $\delta<0.38 \%, \delta<0.36 \%, \delta<1.9 \%$ and $\delta<1.7 \%$ at the $95 \%$ C.I. have been obtained for rigidities above $200 \mathrm{GV}$.

European Physical Society Conference on High Energy Physics - EPS-HEP2019 -

10-17 July, 2019

Ghent, Belgium

* Speaker.

${ }^{\dagger}$ On behalf of the AMS Collaboration 


\section{Introduction}

The precise measurements of the comic ray fluxes performed by AMS have revealed the existence of unexpected structures that can be hardly reconciled with our current understanding of galactic comic ray production and propagation. In particular, the positron spectrum shows distinctive features above $\sim 10 \mathrm{GeV}$ [1], which are not consistent with a pure secondary origin and may indicate the presence of primary sources $[2,3,4]$, whereas protons and nuclei show a progressive hardening above $\sim 100 \mathrm{GeV}[5]$, which may also arise from the presence of local sources [6] or indicate a transition in their propagation regime [7]. In particular, the contribution of compact sources may induce some degree of large scale anisotropy in the arrival directions of the measured cosmic ray fluxes $[8,9]$. Therefore, the determination of the dipole component in galactic coordinates can help understand the origin of the observed features.

\section{AMS detector}

The AMS detector is a large acceptance magnetic spectrometer designed to carry out accurate measurements of charged cosmic rays in the GeV-TeV energy range. It was installed on 19 May 2011 onboard the ISS and has been continuously taking data since then. To date AMS has collected more than $1.4 \times 10^{11}$ events in its long duration mission, which will continue until the end of ISS operations in 2024.

The AMS detector consists of nine layers of precision silicon tracker layers (STD), with an inner tracker (L2-L8) inside a permanent magnet and two outer layers (L1 and L9); a transition radiation detector (TRD); four planes of time of flight counters (TOF); an array of anti-coincidence counters (ACC) surrounding the inner tracker; a ring imaging Čerenkov detector (RICH); and an electromagnetic calorimeter (ECAL). A detailed description of the sub-detectors can be found in [10] and references therein.

\section{Data selection}

The measurement of anisotropies in the arrival directions of electrons and positrons, and protons, carbon and oxygen nuclei was carried out on the data sample corresponding to the first 6.5 and 7.5 years of AMS on the ISS, respectively.

The selection of electrons and positrons follows the quality criteria described in [11] and [12]. Events are selected by requiring a track in the TRD and in the tracker, a cluster of hits in the ECAL, and a measured velocity $\beta \sim 1$ in the TOF consistent with a downward-going $Z=1$ particle. Proton background is reduced below the percent level by means of a cut based selection on TRD and ECAL estimators, and good energy-momentum matching. The remaining sample contains $9.9 \times 10^{4}$ positrons and $1.3 \times 10^{6}$ electrons in the energy range between 16 and $350 \mathrm{GeV}$, with a negligible proton contamination.

The proton and nuclei selections follow the prescriptions in [13] and [14]. The selected samples consist on downward-going particles with a reconstructed track in the inner tracker and an associacted hit in the upstream tracker outer layer L1. For protons, an associated hit in L9 is also required. Finally, a consistent charge determination is required for each particle selection. The selected samples contain $1.3 \times 10^{8}$ protons, $1.0 \times 10^{8}$ helium, $2.9 \times 10^{6}$ carbon and $2.8 \times 10^{6}$ oxygen 
nuclei with reconstructed rigidities above $18 \mathrm{GV}$, with negligible contamination from other cosmic ray species.

In addition, to select only primary cosmic rays, all events are required to be above 1.2 times the maximum local geomagnetic cutoff within the AMS field of view.

\section{Determination of Large Scale Anisotropies}

The analysis of anisotropies in the event sample is performed by comparing the observed distribution of arrival directions in galactic coordinates, $(l, b)$, with a reference map. Skymaps are built using HEALPix scheme [15], which ensures a pixelization with an equal area isolatitude subdivisions of the sphere.

There are two basic approaches to determine the reference maps, which lead to either relative or absolute anisotropy measurements. Unlike relative anisotropies, where a data sample is used as reference, absolute anisotropies require the knowledge of the directional response of the detector. Therefore, computation of isotropic skymaps for absolute anisotropies requires detailed understanding of the detector. Systematic procedures, valid for all cosmic ray species, have been developed to obtain these isotropic skymaps [16, 17].

The directional flux, resulting from the ratio of data over reference skymaps, is described by means of an spherical harmonic expansion

$$
\Phi(l, b)=\Phi_{0}\left(1+\sum_{\ell>0} \sum_{m=-\ell}^{m=+\ell} a_{\ell m} Y_{\ell m}(l, b)\right)
$$

where the multipolar coefficients, $a_{\ell m}$, are determined from a likelihood fit.

The dipole component, $\ell=1$, is fully described by three orthonormal functions corresponding to three orthogonal axes: $Y_{1+1}$ is aligned with the forward-backward direction, pointing to the galactic center; $Y_{1+0}$ is aligned with the north-south direction, pointing to the north galactic pole; and $Y_{1-1}$ is aligned with the east-west direction, contained in the galactic plane and completes the right-handed coordinate system. Dipole components in each direction are defined as

$$
\rho_{E W}=\sqrt{\frac{3}{4 \pi}} a_{1-1} \quad ; \quad \rho_{N S}=\sqrt{\frac{3}{4 \pi}} a_{1+0} \quad ; \quad \rho_{F B}=\sqrt{\frac{3}{4 \pi}} a_{1+1} .
$$

This allows to define the dipole amplitude as

$$
\delta=\sqrt{\rho_{E W}^{2}+\rho_{N S}^{2}+\rho_{F B}^{2}} .
$$

\section{Results}

The absolute anisotropy of the positron and electron samples is determined in 5 cumulative energy ranges, with minimum energies of $16,25,40,65$ and $100 \mathrm{GeV}$ and a maximum energy of $350 \mathrm{GeV}$ according to their measured energy in the ECAL. Likewise, the measurement of the absolute anisotropy of the proton and nuclei samples is performed in 9 reconstructed rigidity bins, with minimum rigidities ranging from 18 to $1000 \mathrm{GV}$. 
For each particle species and energy range, the small geographical dependence of the detector efficiencies is estimated directly on data and introduced as a correction to its reference skymap. The validity of these corrections is verified with the determination of the amplitude of the multipolar expansion in geographic coordinates. The projection of the residual contributions into the galactic coordinate system is taken as a systematic uncertainty of the measurement. This uncertainty, together with the statistical error of the efficiency corrections constitute the dominant contribution to the systematic errors.

The amplitude of the dipole components in galactic coordinates is found to be consistent with isotropy for all particle species and energy ranges. The uncertainty of the measurement is dominated by the finite statistics of the selected data sample for positron, electrons and nuclei in the complete energy range, and for protons above $70 \mathrm{GV}$. At lower rigidities, the systematics on the efficiency corrections limit the sensitivity to the per mil level.

Since no significant deviation from isotropy is observed, upper limits to the dipole amplitude for each particle species and energy range have been derived following the procedure described in [18], and are displayed in Figs. 1, 2 and 3. In particular, for positrons and electrons above $16 \mathrm{GeV}$, $\delta<1.9 \%$ and $\delta<0.5 \%$ at the $95 \%$ C.I. For protons, helium, carbon and oxygen above $200 \mathrm{GV}$, $\delta<0.38 \%, \delta<0.36 \%, \delta<1.9 \%$ and $\delta<1.7 \%$ at the $95 \%$ C.I., respectively.

The absence of significant dipole anisotropy in positrons above $\sim 10 \mathrm{GeV}$ can be used to constrain source models. In this sense, by the end of its operation on the ISS, AMS expects to reach the sensitivity to test the pulsar origin of this excess. The results for electrons, where the relative source contribution and thus the expected anisotropy should be much smaller, confirm the absence of systematics in this measurement beyond the few per mil level. On the other hand, the absence of large dipole components in protons and nuclei above $\sim 100 \mathrm{GeV}$ can be used to constrain models that make use of local sources to explain the spectral hardening observed by AMS. Finally, the results obtained on both protons and electrons confirm that the anisotropy of the diffuse component of cosmic rays is below the per mil level in the energy range where the positron excess is observed and, in particular, below the expectation from pulsar models for its origin.
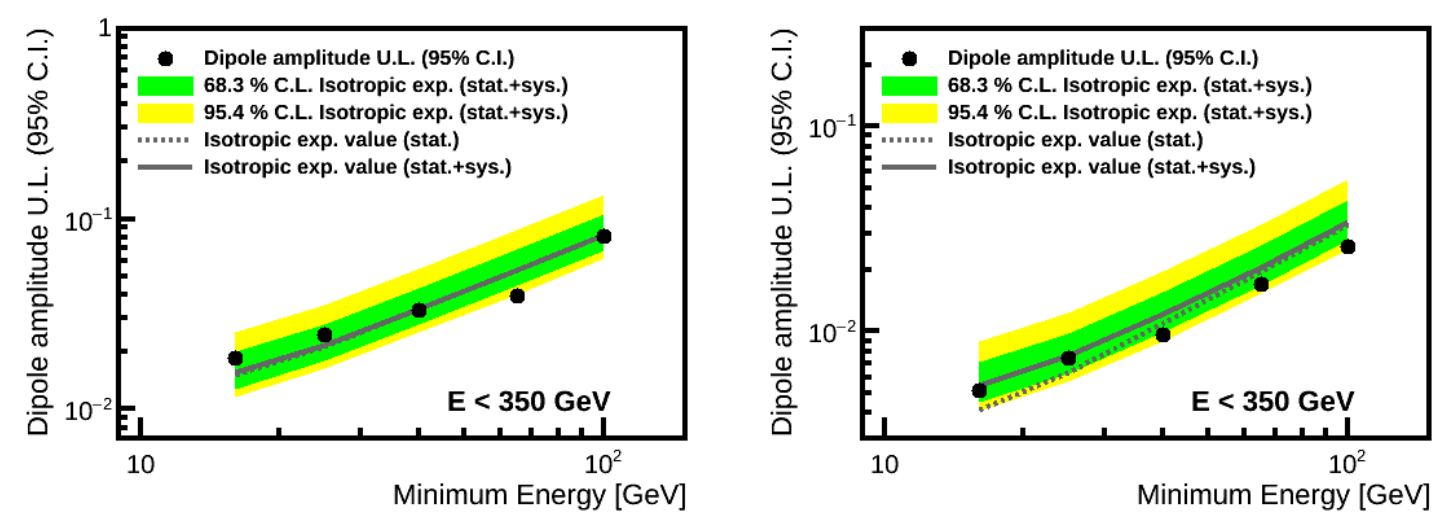

Figure 1: Upper limits on the dipole amplitudes of positrons (left) and electrons (right). The points correspond to the AMS measurement. The solid and dashed lines correspond to the isotropic expectation with or without including the systematic uncertainties of the measurement. Bands corresponding to the $68.3 \%$ and $95.4 \%$ C.L. are also displayed. 

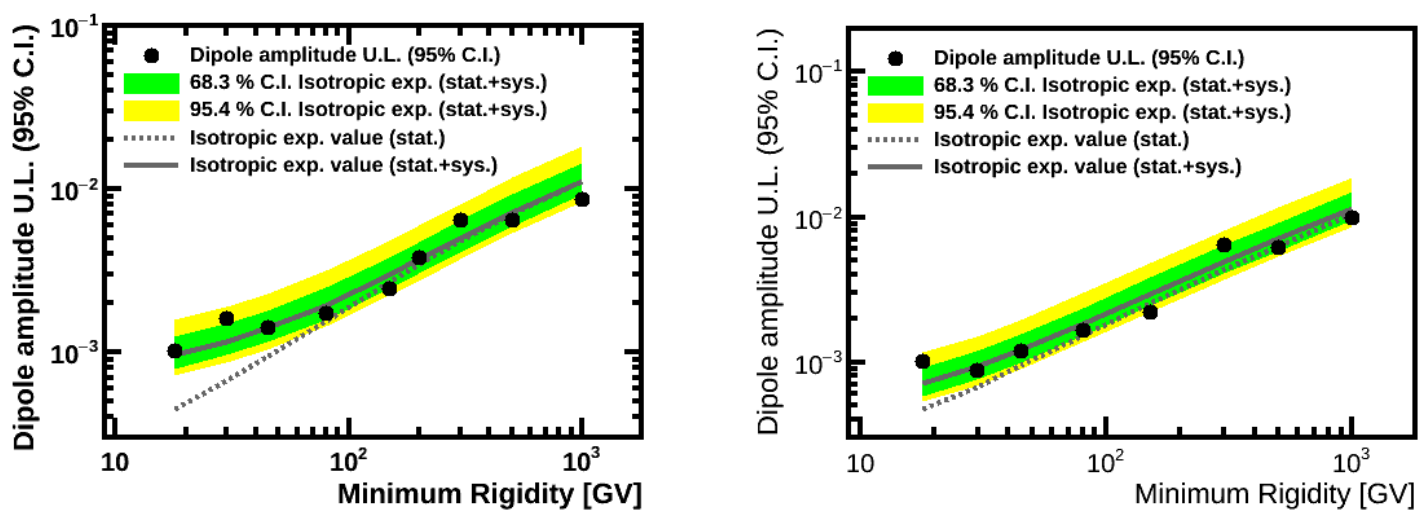

Figure 2: Upper limits on the dipole amplitudes of protons (left) and helium (right). The points correspond to the AMS measurement. The solid and dashed lines correspond to the isotropic expectation with or without including the systematic uncertainties of the measurement. Bands corresponding to the $68.3 \%$ and $95.4 \%$ C.L. are also displayed.
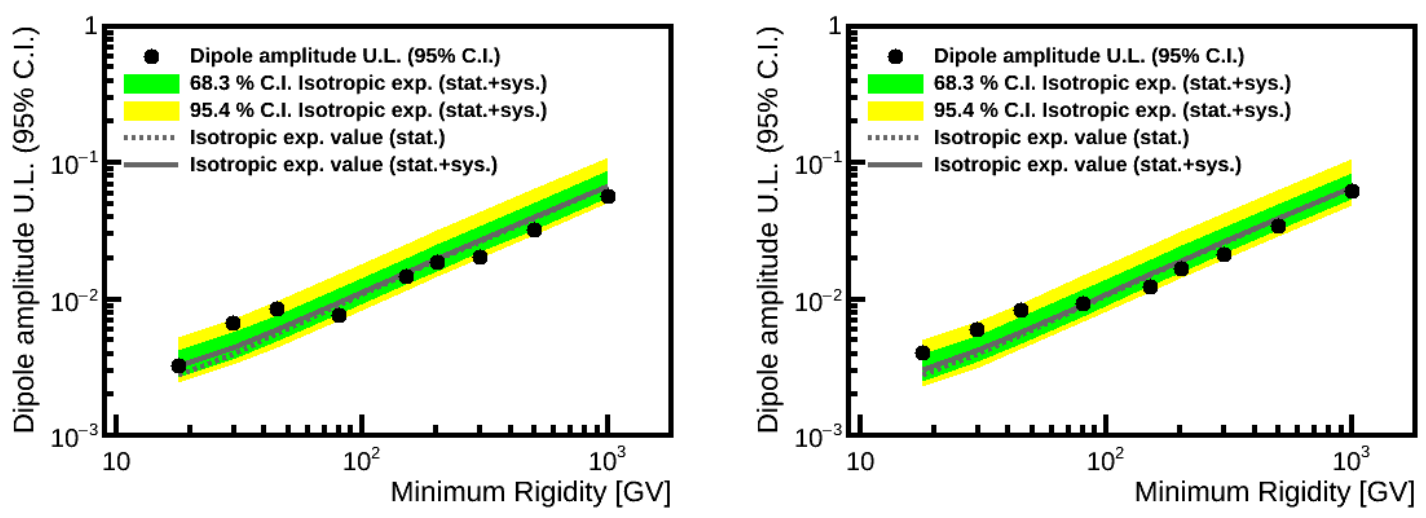

Figure 3: Upper limits on the dipole amplitudes of carbon (left) and oxygen (right). The points correspond to the AMS measurement. The solid and dashed lines correspond to the isotropic expectation with or without including the systematic uncertainties of the measurement. Bands corresponding to the $68.3 \%$ and $95.4 \%$ C.L. are also displayed.

\section{References}

[1] A. Kounine [AMS Collaboration], Latest results from the AMS Experiment, in these proceedings.

[2] P. D. Serpico, Astrophysical models for the origin of the positron "excess", Astropart.Phys. 39-40, 2 (2012) [arXiv:1108.4827 [astro-ph.HE]].

[3] M. Boudaud et al., A new look at the cosmic ray positron fraction, Astronomy \& Astrophysics, 575, A67 (2015) [arXiv:1410.3799 [astro-ph.HE]] 
[4] M. Di Mauro, F. Donato, N. Fornengo, A Vittino, Dark matter vs. astrophysics in the interpretation of AMS-02 electron and positron data, JCAP, 1605, 031 (2016) [arXiv: 1507.07001 [astro-ph. HE]]

[5] L. Derome [AMS Collaboration], Properties of Primary and Secondary Cosmic Ray Nuclei Measured with AMSO2, in these proceedings.

[6] P. Blasi, E. Amato, Diffusive propagation of cosmic rays from supernova remnants in the Galaxy. II: anisotropy, JCAP, 2012, 011 (2012) [arXiv:1105.4529 [astro-ph.HE]].

[7] P. Blasi, E. Amato, P. D. Serpico, Spectral breaks as a signature of cosmic ray induced turbulence in the Galaxy, Phys.Rev.Lett. 109, 109, 061101 (2012) [arXiv:1207.3706 [astro-ph. HE] ].

[8] I. Cernuda, Cosmic-ray electron anisotropies as a tool to discriminate between exotic and astrophysical sources, Astropart.Phys., 34, 59 (2010) [arXiv:0905.1653 [astro-ph. HE] ].

[9] S. Manconi, M. Di Mauro, F. Donato, Dipole anisotropy in cosmic electrons and positrons: inspection on local sources, JCAP, 1701, 006 (2017) [arXiv: 1611.06237 [astro-ph.HE] ].

[10] M. Aguilar et al. [AMS Collaboration], First Result from the Alpha Magnetic Spectrometer on the International Space Station: Precision Measurement of the Positron Fraction in Primary Cosmic Rays of 0.5-350 GeV, Phys.Rev.Lett. 110, 141102 (2013).

[11] M. Aguilar et al. et al. [AMS Collaboration], Towards Understanding the Origin of Cosmic-Ray Electrons, Phys.Rev.Lett. 122, 101101 (2019).

[12] M. Aguilar et al. [AMS Collaboration], Towards Understanding the Origin of Cosmic-Ray Positrons, Phys.Rev.Lett. 122, 041102 (2019).

[13] M. Aguilar et al. [AMS Collaboration], Precision Measurement of the Proton Flux in Primary Cosmic Rays from Rigidity $1 \mathrm{GV}$ to $1.8 \mathrm{TV}$ with the Alpha Magnetic Spectrometer on the International Space Station, Phys.Rev.Lett. 114, 171103 (2015).

[14] M. Aguilar et al. [AMS Collaboration], Observation of the Identical Rigidity Dependence of He, C, and $O$ Cosmic Rays at High Rigidities by the Alpha Magnetic Spectrometer on the International Space Station, Phys.Rev.Lett. 119, 251101 (2017).

[15] K. M. Górski et al., HEALPix: A Framework for High-Resolution Discretization and Fast Analysis of Data Distributed on the Sphere, Astrophys.J. 622, 759 (2005) [arXiv: astro-ph/ 0409513 [astro-ph]].

[16] M.A. Velasco, Ph.D. thesis, Universidad Complutense de Madrid (2018), [https: //cds.cern.ch/record/2640369].

[17] S. Zeissler, Ph.D. thesis, Karlsruher Institut für Technologie (2018), [http://ekp-invenio.physik.uni-karlsruhe.de/record/48952].

[18] C. Maña. Probability and Statistics for Particle Physics. UNITEXT for Physics. Springer International Publishing, 2017. ISBN 9783319557380. 